\title{
Immune signature-based risk stratification and prediction of immune checkpoint inhibitor's efficacy for lung adenocarcinoma
}

\author{
Ming $\mathrm{Yi}^{1} \cdot$ Anping $\mathrm{Li}^{2} \cdot$ Linghui Zhou ${ }^{3} \cdot$ Qian $\mathrm{Chu}^{2} \cdot$ Suxia $\mathrm{Luo}^{2} \cdot \mathrm{Kongming} \mathrm{Wu}^{1,2}$ (])
}

Received: 13 June 2020 / Accepted: 1 December 2020 / Published online: 2 January 2021

(c) The Author(s) 2021

\begin{abstract}
Background Lung adenocarcinoma (LUAD) is a common pulmonary malignant disease with a poor prognosis. There were limited studies investigating the influences of the tumor immune microenvironment on LUAD patients' survival and response to immune checkpoint inhibitors (ICIs).

Methods Based on TCGA-LUAD dataset, we constructed a prognostic immune signature and validated its predictive capability in the internal as well as total datasets. Then, we explored the differences of tumor-infiltrating lymphocytes, tumor mutation burden, and patients' response to ICI treatment between the high-risk score group and low-risk score group.

Results This immune signature consisted of 17 immune-related genes, which was an independent prognostic factor for LUAD patients. In the low-risk score group, patients had better overall survival. Although the differences were non-significant, patients with low-risk scores had more tumor-infiltrating follicular helper T cells and fewer macrophages (M0), which were closely related to clinical outcomes. Additionally, the total TMB was markedly decreased in the low-risk score group. Using immunophenoscore as a surrogate of ICI response, we found that patients with low-risk scores had significantly higher immunophenoscore.
\end{abstract}

Conclusion The 17-immune-related genes signature may have prognostic and predictive relevance with ICI therapy but needs prospective validation.

Keywords Lung adenocarcinoma $\cdot$ Immunotherapy $\cdot$ The tumor immune microenvironment $\cdot$ Immune checkpoint inhibitor · Tumor mutation burden · Prognostic model

\section{Abbreviations}

ALK Anaplastic lymphoma kinase

B2-AR $\quad \beta 2$-Adrenergic receptor

CTLA-4 Cytotoxic T-lymphocyte-associated antigen 4

EGFR Epidermal growth factor receptor

GO Gene ontology

KEGG Kyoto Encyclopedia of Genes and Genomes
ICI Immune checkpoint inhibitor

IRG Immune-related gene

LASSO Least absolute shrinkage and selection operator

LUAD Lung adenocarcinoma

MDSC Myeloid-derived suppressor cell

NSCLC Non-small cell lung cancer

PD-1 Programmed cell death 1
Suxia Luo

luosxrm@163.com

Kongming Wu

kmwu@tjh.tjmu.edu.cn

Ming Yi

1978135000@qq.com

Anping Li

li_anping@yahoo.com

Linghui Zhou

2544928065@qq.com

Qian Chu

qianchu@tjh.tjmu.edu.cn
1 Department of Oncology, Tongji Hospital of Tongji Medical College, Huazhong University of Science and Technology, Wuhan 430030, China

2 Department of Medical Oncology, The Affiliated Cancer Hospital of Zhengzhou University and Henan Cancer Hospital, Zhengzhou 450008, China

3 Bone Marrow Transplantation Center, The First Affiliated Hospital, Zhejiang University School of Medicine, No. 79 Qingchun Road, Hangzhou 310003, China 


$\begin{array}{ll}\text { PD-L1 } & \text { Programmed cell death-ligand } 1 \\ \text { TAM } & \text { Tumor-associated macrophage } \\ \text { TCGA } & \text { The Cancer Genome Atlas } \\ \text { TLR8 } & \text { Toll-like receptor } 8 \\ \text { TMB } & \text { Tumor mutation burden } \\ \text { Treg } & \text { Regulatory T cell }\end{array}$

\section{Background}

In the United States, lung cancer is the first leading cause of cancer-related deaths in 2019 [1]. According to histology, lung cancer could be classified into small cell lung cancer (15\% of all cases) and non-small cell lung cancer (NSCLC) ( $85 \%$ of all patients). NSCLC is further divided into three subtypes: adenocarcinoma, squamous carcinoma, and large cell carcinoma. Among these three subtypes, lung adenocarcinoma (LUAD) is most common and accounts for approximately $40 \%$ of lung cancer [2]. In the past decades, due to the advances of cancer genomics, a group of gene alterations is identified as driver gene mutations for LUAD, such as mutations in epidermal growth factor receptor $(E G F R)$, c-MET, KRAS, anaplastic lymphoma kinase $(A L K)$ [3-7]. Then, multiple agents targeting driver gene mutations have been developed. Unfortunately, after receiving targeting treatments, such as EGFR-tyrosine kinase inhibitors, most patients eventually become resistant to targeting therapy, partly because of secondary mutations in tumors [5].

Cancer immunology and immunotherapy provide a novel perspective for cancer therapeutics [8]. LUAD tends to have a high tumor mutation burden (TMB) and strong immunogenicity [9]. Therefore, LUAD is an ideal indication of immunotherapy [9]. In the clinic, immune checkpoint inhibitors (ICIs) targeting programmed cell death 1 (PD-1) and programmed cell death-ligand 1 (PD-L1) exhibit a potent and durable anti-tumor activity in LUAD patients [10]. However, the overall response rate of ICI is relatively low, and only a subset of LUAD patients could benefit from ICI treatment [11]. Up to now, a series of biomarkers have been verified to herald the efficacy of ICI treatment including TMB, PD-L1 expression level, neoantigens, gut microbiota, the status of immune cells [8]. Generally, most biomarkers reflect the status of the tumor immune microenvironment in a certain aspect. Recently, some computer algorithms, such as TIMER and CIBERSORT, make it feasible to estimate the immune profiling of cancer by transcriptome sequencing files $[12,13]$. Constructing a comprehensive immune profiling-based model would be meaningful to predict the efficacy of ICI [14].

Actually, the immune landscape of cancer heralds the effect of immunotherapy and closely relates to patients' prognosis [15]. Multiple previous studies indicated that some immune-related genes (IRGs) are prognostic biomarkers for colorectal cancer, ovarian cancer, and hepatocellular carcinoma [16-18]. There are few studies investigating the predictive value of IRGs and immune profiling in LUAD. In this study, we constructed an immune signature based on IRGs and explored the relationship between this immune signature and LUAD patients' clinic-pathologic features as well as clinical outcomes. Additionally, we mapped the immune landscape, analyzed TMB, and predicted the response to ICI treatment in patients with different risk scores.

\section{Materials and methods}

\section{Data acquiring and cleaning}

LUAD patients' transcriptome sequencing data and clinical information were download from UCSC Xena the Cancer Genome Atlas (TCGA) LUAD cohort (https://xenabrowse r.net/). The mutation profiling was acquired from TCGA data portal (https://portal.gdc.cancer.gov/) by R software (version: 4.0.0) with package TCGAbiolinks [19]. Data cleaning was conducted by R software. The list of IRGs was downloaded from ImmPort database (https://immport.niaid.nih. gov) [20].

\section{Differentially expressed genes}

To screen out the IRGs participating in the oncogenesis, we analyzed the differentially expressed genes between LUAD and corresponding normal tissues. By package edgeR, abnormally expressed genes were selected as previously described (adjusted $p$ value $<0.05$ and $\log 2$ (fold change) $\mid>1$ ) [21]. Differentially expressed IRGs were the intersection between IRGs and differentially expressed genes.

\section{Pathway and function enrichment analysis}

To explore the biological significances of these differentially expressed IRGs, we performed pathway and function enrichment analysis with the online tool DAVID Bioinformatics Resources 6.8 (https://david.ncifcrf.gov/). Kyoto Encyclopedia of Genes and Genomes (KEGG) and gene ontology (GO) enrichment analyses were performed. Pathways and terms with false discovery rate $<0.05$ were considered as significantly enriched objects. The visualization was performed by package ggplot 2 .

\section{Constructing an IRG-related immune signature for LUAD}

The TCGA LUAD patients were randomly divided into a training set ( $2 / 3$ for all patients) and a test set (1/3 for all 
patients). We used the training set to identify prognosisrelated immune genes and constructed a prognostic risk model. Then, the predictive power and robustness of the model were validated by the test set and total patients. We first screened out prognosis-related immune genes by univariate Cox proportional hazard regression. To avoid overfitting, all genes with $p$ value $<0.05$ were involved in the subsequent least absolute shrinkage and selection operator (LASSO) analysis with package glmnet. After the filtration by LASSO model, the selected genes were used to construct the immune-related risk model by multivariate Cox proportional hazards model: risk score $=$ level of gene $a *$ coefficient $a+$ level of gene $b *$ coefficient $b+$ level of gene $c *$ coefficient $c+\ldots \ldots+$ level of gene $n *$ coefficient $n$ [15]. In the model, the risk score reflected the prognosis of LUAD patients: the lower the score, the better the prognosis. Setting the median of risk score as the cutoff value, the patients were classified into a high-risk group and a low-risk group. The predictive power was calculated by Kaplan-Meier survival curves, and log-rank $p$ value $<0.05$ was regarded as statistically significant (with packages survival and survminer). To assess the predictive capability of this immune signature, time-dependent receiver operating characteristic curves were used (package survivalROC).

\section{Calculating the ratio of tumor-infiltrating immune cells}

CIBERSORT could calculate the ratios of infiltrated immune cells from tissue transcriptional profiles by a deconvolution algorithm [13]. Based on TCGA LUAD transcriptional profiles and R script of CIBERSORT, we calculated the ratios of 22 types of tumor-infiltrating immune cells.

\section{TMB analysis}

LUAD patients' somatic variants data were analyzed and visualized by package maftools (the pipeline of MAF file: muse) [22]. Then, we calculated the TMB of each patient (mutations per million bases).

\section{Predicting the patients' response to ICI}

The Cancer Immunome Atlas (https://tcia.at/) analyzed the immune landscapes and antigenomes of 20 solid tumors. Tumor immunogenicity was quantitatively scored from 0 to 10, which was termed immunophenoscore (IPS) [23]. The IPS value was positively correlated to tumor immunogenicity. It has been verified that IPS could predict the patients' response to ICI treatment [23]. We extracted data of IPS for the following analysis. Additionally, we compared the mRNA levels of immune checkpoints and their ligands in high-risk score group and low-risk score group.

\section{Statistical analysis}

Differences among variables were tested by Student's $t$ test and Chi square test. Univariate and multivariate cox regression analyses were used to assess the influences of the immune signature and multiple clinic-pathological parameters on patients' survival. Statistical analysis was performed by R software (4.0.0). The heat maps were plotted by package pheatmap. A two-sided $p<0.05$ was regarded as statistically significant.

\section{Results}

\section{The characteristics of patients}

RNA-sequencing profiles and clinic-pathological data of 497 LUAD patients were downloaded from UCSC Xena TCGA-LUAD dataset. Patients were randomly divided into a training set (331 patients) and a test set (166 patients). For most clinic-pathological parameters, there was no significant difference between the training set and test set (Table 1).

\section{Identifying differentially expressed genes}

With the cutoff value adjusted $p<0.05$ and $\mid \log 2$ (fold change) $\mid>1,783$ differentially expressed genes were screened out. Among them, 545 genes were upregulated, while 238 genes were downregulated in tumors (Fig. 1a). Based on the list of differentially expressed genes, 88 differentially expressed IRGs were selected (Fig. 1b). Pathway and function enrichment analyses were performed by the online annotation tool DAVID. The top five most enriched KEGG pathways were: cytokine-cytokine receptor interaction, neuroactive ligand-receptor interaction, regulation of lipolysis in adipocytes, PI3K-Akt signaling pathway, and rheumatoid arthritis (Fig. 1c). The top five most enriched GO- molecular function terms were: hormone activity, heparin binding, growth factor activity, cytokine activity, and chemokine activity (Fig. 1d).

\section{A 17-gene immune signature can predict the prognosis of LUAD patients}

To explore the prognostic value of selected IRGs, we conducted a univariate Cox regression analysis. 32 genes were significantly associated to the overall survival (OS) in the training set. To avoid overfitting, we further conducted a LASSO analysis and 17 of 32 genes were predictors for patients' prognosis (Fig. 2a-b). Then, based on the training set, we conducted a multivariate Cox proportional hazards regression analysis and established a predictive model: risk score $=\left(V I P R I{ }^{*}-0.08876\right)+(B I R$ 
Table 1 The clinical characteristics of LUAD patients

\begin{tabular}{|c|c|c|c|c|c|}
\hline Variables & Group & Total set $(n=497)$ & $\begin{array}{l}\text { Training set } \\
(n=331)\end{array}$ & Test set $(n=166)$ & $P$ value \\
\hline \multirow[t]{2}{*}{ Vital status } & Alive & 317 & 206 & 111 & \multirow[t]{2}{*}{0.36} \\
\hline & Dead & 180 & 125 & 55 & \\
\hline Survival time & & 911.24 & 898.1 & 937.43 & 0.66 \\
\hline \multirow[t]{5}{*}{ Clinical stage } & I & 267 & 187 & 80 & \multirow[t]{5}{*}{0.17} \\
\hline & II & 118 & 75 & 43 & \\
\hline & III & 80 & 48 & 32 & \\
\hline & IV & 25 & 17 & 8 & \\
\hline & NA & 7 & 4 & 3 & \\
\hline \multirow[t]{3}{*}{ T stage } & $\mathrm{T} 1-\mathrm{T} 2$ & 433 & 288 & 145 & \multirow[t]{3}{*}{0.64} \\
\hline & T3-T4 & 61 & 43 & 18 & \\
\hline & NA & 3 & 0 & 3 & \\
\hline \multirow[t]{3}{*}{$\mathrm{N}$ stage } & No & 321 & 223 & 98 & \multirow[t]{3}{*}{0.11} \\
\hline & N1-N3 & 165 & 102 & 63 & \\
\hline & NA & 11 & 6 & 5 & \\
\hline \multirow[t]{3}{*}{ M stage } & M0 & 331 & 226 & 105 & \multirow[t]{3}{*}{0.97} \\
\hline & M1 & 24 & 17 & 7 & \\
\hline & NA & 142 & 88 & 54 & \\
\hline \multirow[t]{2}{*}{ Age } & $<65$ & 218 & 142 & 76 & \multirow[t]{2}{*}{0.61} \\
\hline & $\geq 65$ & 279 & 189 & 90 & \\
\hline
\end{tabular}

$N A$ not available

Patients with not available data were excluded in statistical analysis
$C 5 * 0.16676)+(G D F 10 * 0.12945)+(A D R B 2 * 0.03706)$ $+\left(I L 20 R B^{*} 0.06621\right)+\left(L G R 4^{*} 0.15962\right)+\left(I N H A^{*} 0.062\right.$ $04)+(C D 19 * 0.01529)+\left(S 100 P^{*} 0.04467\right)+(I G K V 1.8 *$ $0.05711)+\left(I G K V 1 D .43 *_{-0.02639)}+\left(A D R B 1 *_{-}\right.\right.$ $0.30831)+(H T R 3 A * 0.01446)+(A D M 2 *$ $0.09179)+(T L R 8 *-0.19053)+$ $(G R E M 1 * 0.08239)+($ IGHV3.64*-0.10157) (Fig. 2c) Seven genes were protective factors (IGKVID.43, IGKVI.8, VIPR1, ADM2, IGHV3.64, TLR8, and ADRB1) while ten genes were risk factors (BIRC5, LGR4, GDF10, GREM1, IL2ORB, INHA, S100P, ADRB2, CD19, and HTR3A).

According to the formula mentioned above, the risk score of each patient in the training set was calculated. Patients were classified into the high-risk group and lowrisk group using the median risk score as the cutoff value. Patients in the high-risk group had a significantly poorer OS $(P<0.0001)$ (Fig. 3a). The areas under the curves (AUCs) of this immune signature were 0.74 for 3 -year OS and 0.70 for 5-year OS (Fig. 3b). Besides, we ranked patients' risk scores and overviewed their distributions (Fig. 3c). Patients' risk scores and survival times were presented in the dot plot (Fig. 3d). The heat map exhibited the expression patterns of 17 IRGs in patients with different risk scores (Fig. 3e).

The predictive capability of this immune signature was further verified in the testing set. According to the predictive signature, each patient in the testing set was divided into the high-risk group and low-risk group as previously described.
Survival analysis showed that patients with low-risk scores had longer OS ( $p=0.039$ ) (Fig. 4a). The AUC of 3-year OS was 0.58 , and the AUC of 5-year OS was 0.52 (Fig. 4b). The distribution of risk score, patients' survival status, and survival were presented by scatter plots (Fig. 4c-d). The expression of 17 selected genes was visualized by heat map (Fig. 4e).

Besides, the robustness of the 17-IRG signature was assessed in the total set. The high-risk score was an unfavorable factor of LUAD patients $(p<0.0001)$ (Fig. 5a). The AUC of 3-year OS was 0.69, and the AUC of 5-year OS was 0.64 (Fig. 5b). The distribution of risk score, patients' vital status, OS time, and 17 IRGs expression were also presented in Fig. 5c-e.

\section{The 17-gene immune signature is an independent prognostic factor for LUAD patients}

We performed a univariate Cox regression analysis to assess the influences of patients' clinic-pathological factors and immune signature risk score on patients' OS in the total set (Table 2). The results indicated that bigger tumor size, lymph node metastasis, distant metastasis, advanced TNM stage, and high-risk score were unfavorable factors for OS. Then, we performed a multivariate Cox regression analysis. It was found that the risk score of the 17-IRG signature 
A

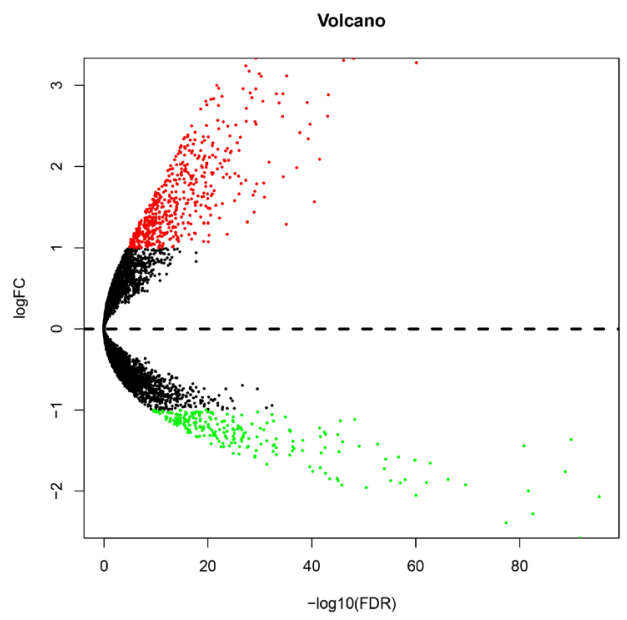

C

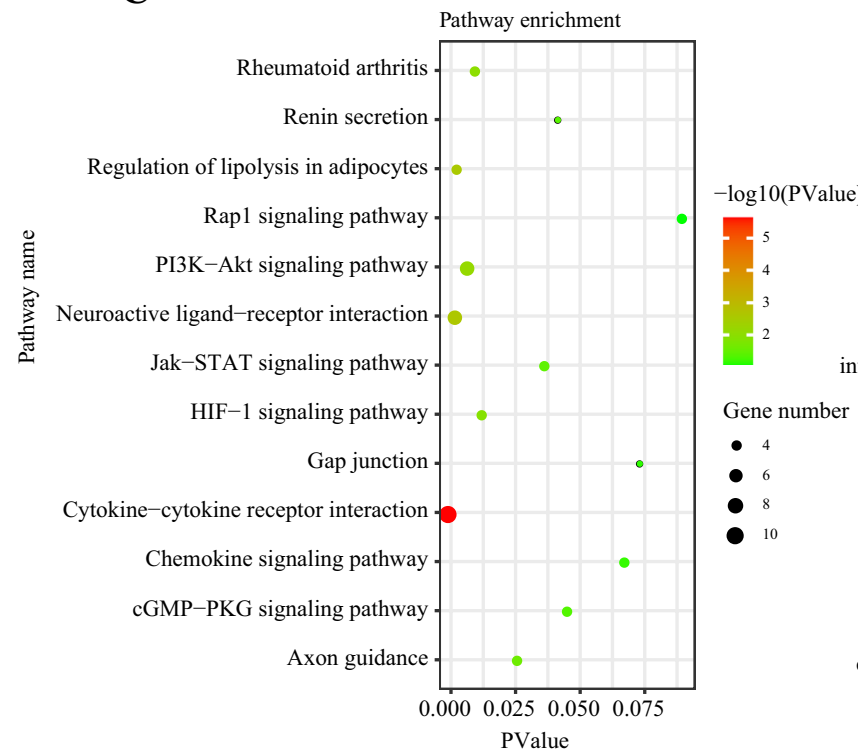

Fig. 1 Identifying differentially expressed immune-related genes and enrichment analyses. a Volcano plot of differentially expressed genes between tumors and normal lung tissues. b Venn diagram of the intersection between differentially expressed genes and immune-related

was an independent prognostic factor $(\mathrm{HR}=2.2,95 \% \mathrm{CI}$ $1.51 \sim 3.2 ; p<0.0001)$.

\section{The associations between 17-IRG signature and patients' clinic-pathological parameters}

We analyzed the relationships between this 17-IRG signature and patients' clinic-pathological parameters, including tumor burden, tumor size, lymph node status, distant metastasis, TNM stage, and age at diagnosis (Fig. 6a-f). The results showed that this 17 -IRG risk score was significantly

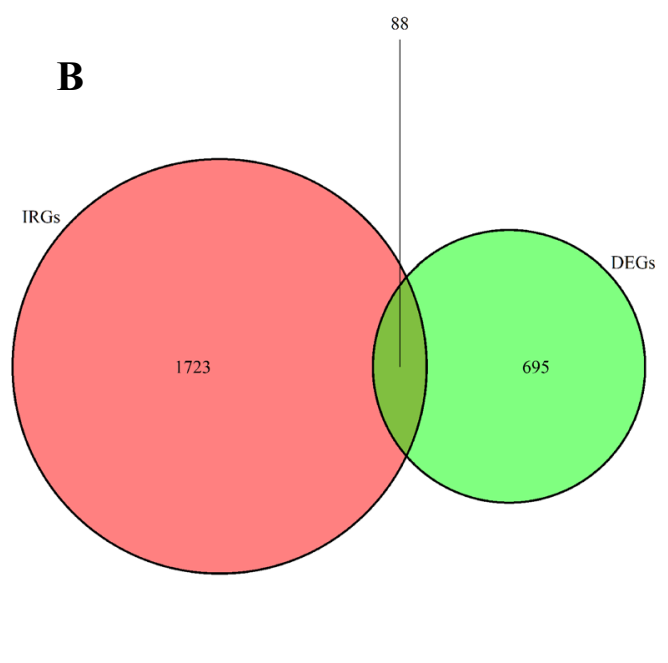

D

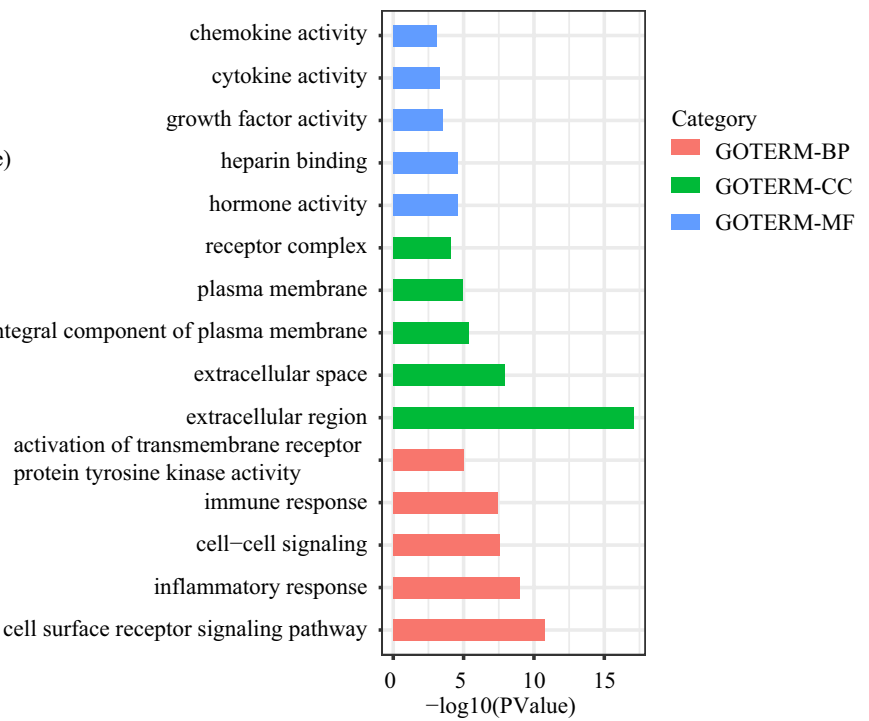

genes. c KEGG pathway enrichment analysis. d Gene ontology enrichment analysis. $B P$ biology process; $C C$ cellular component; $M F$ molecular function

higher in patients with higher tumor burden, bigger tumor size, lymph node metastasis, advanced TNM stage, and age below 65 . Besides, the risk score was higher in patients with distant metastasis, and the difference was on the verge of statistical significance $(p=0.070)$.

\section{The 17-IRG signature and tumor immune microenvironment}

Based on the CIBERSORT algorithm, we calculated the proportions of 22 types of immune cells in each LUAD 
A

32313131302927252321191817171414963

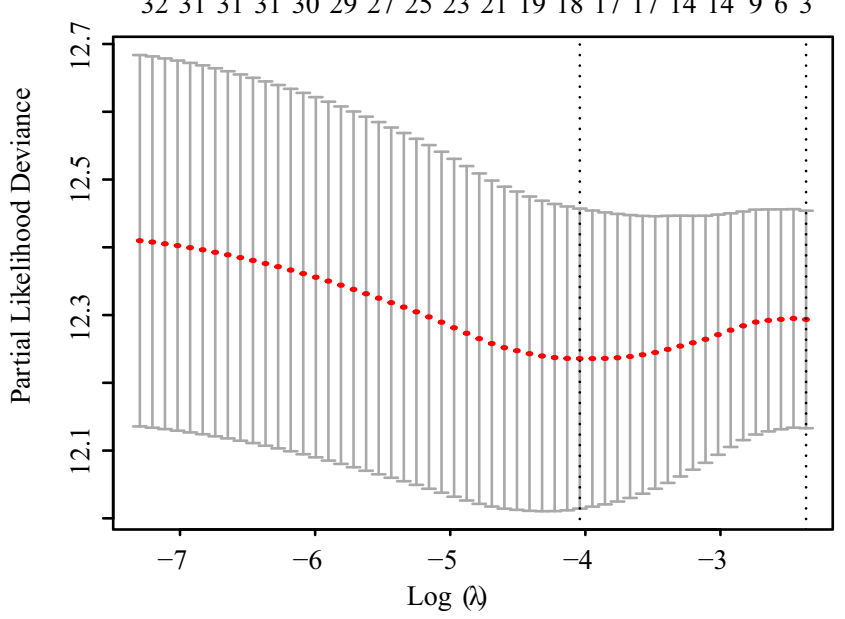

C

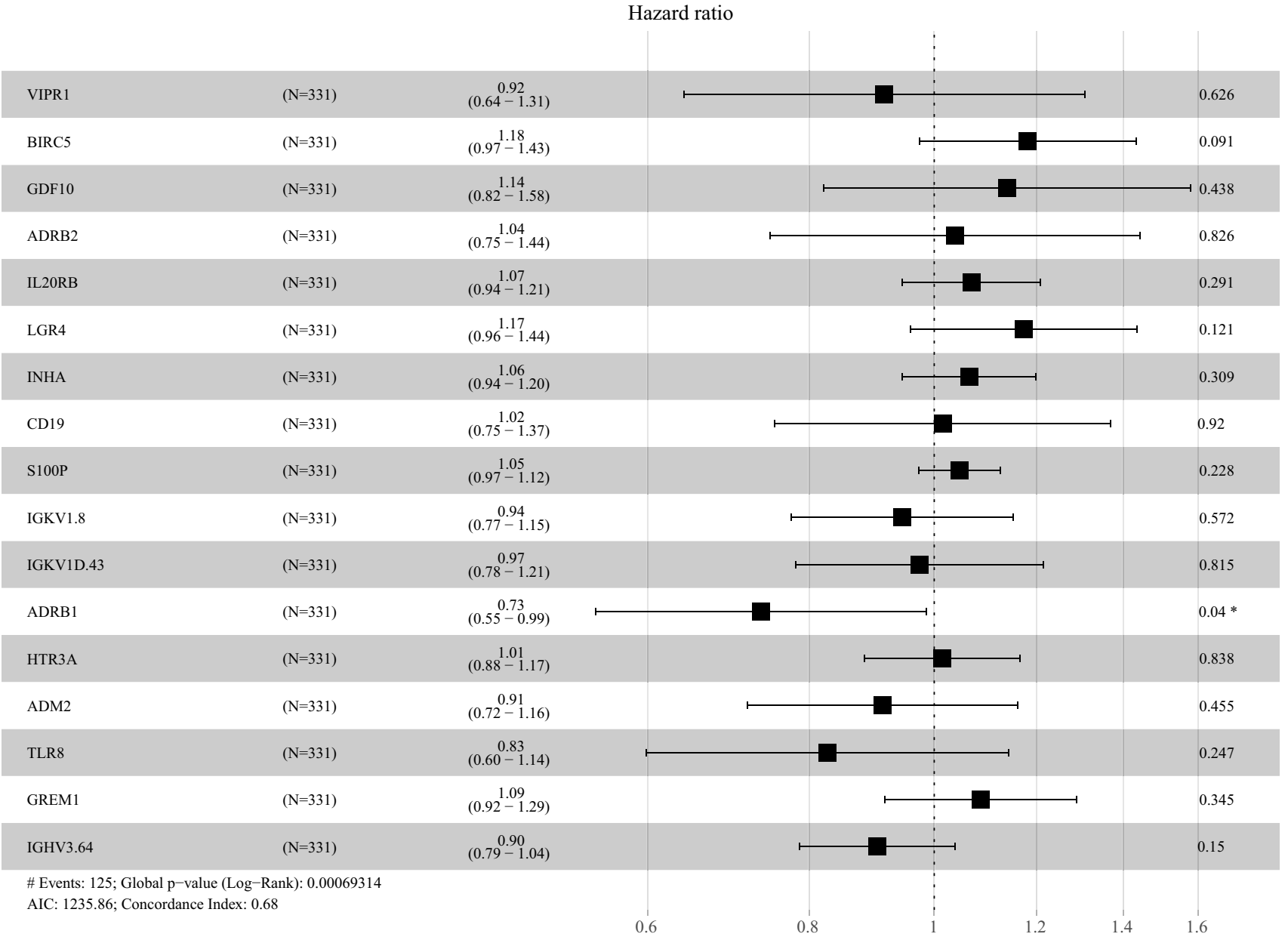

Fig. 2 LASSO analysis and forest plot presenting the multivariable Cox model results of 17 immune-related genes. a cross-validation for tuning parameter selection in the LASSO model. b LASSO coef-
B

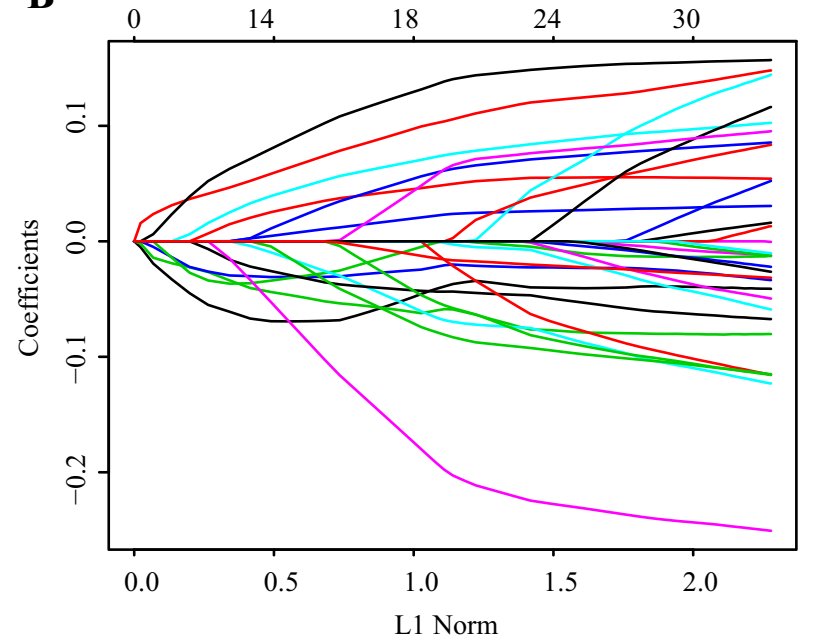

azard ratio 


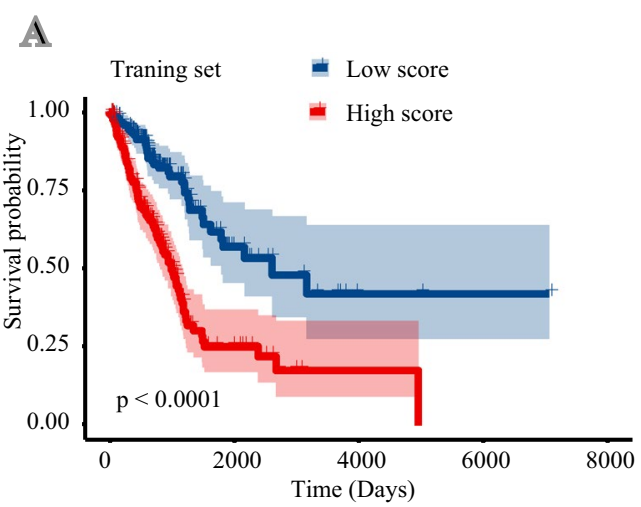

C

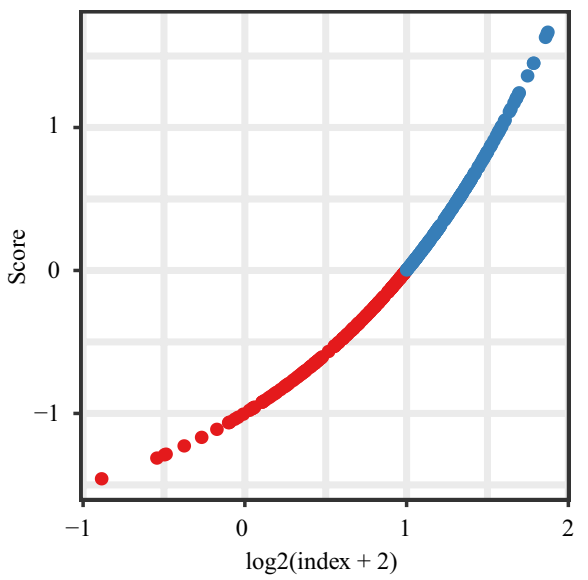

B

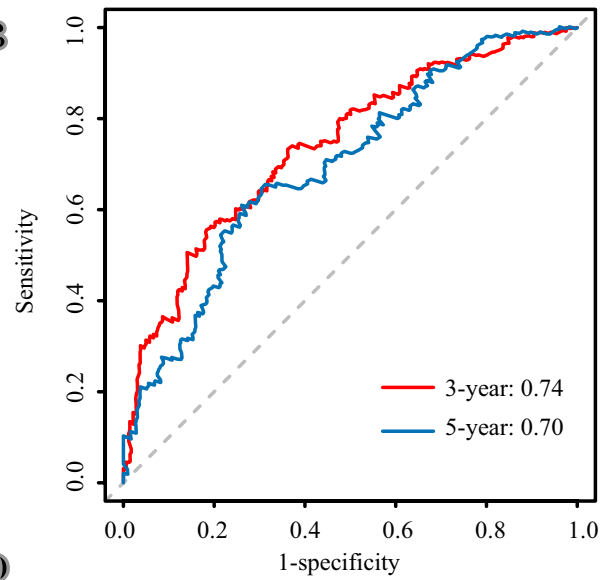

Low score

- High score

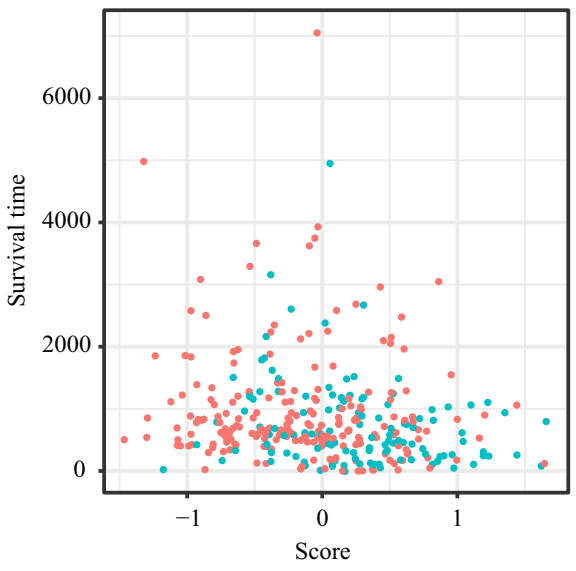

- Alive

- Dead
$\mathbf{E}$

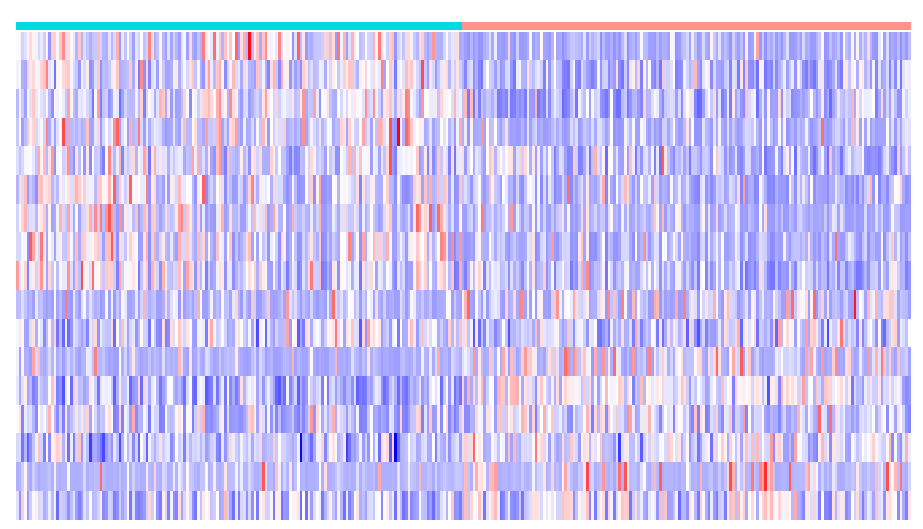

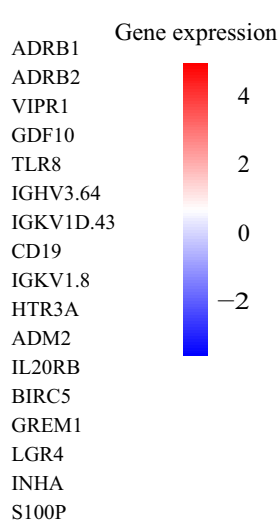

Annotation

Low score

High score

Fig. 3 Constructing a 17-immune-related gene signature in the training set. a Kaplan-Meier curves of overall survival of LUAD patients in high- and low-risk score groups. b Time-dependent receiver operating characteristic curves. $\mathbf{c}$ The distribution of risk scores. $\mathbf{d}$ The

sample. Then, we compared the differences in proportions of immune cells between the high-risk score group and lowrisk score group. It was found that the ratios of plasma cell, monocyte, and resting mast cell were significantly higher in the low-risk score group. On the contrary, the proportions of activated memory $\mathrm{CD} 4^{+} \mathrm{T}$ cell, resting NK cell, relationship between risk scores and survival times. e The expression patterns of 17 immune-related genes in high- and low-risk score groups

macrophage (M0), activated dendritic cell, activated mast cell, and neutrophil were significantly higher in the highrisk score group (Fig. 7a). In 22 types of immune cells, high macrophage (M0) level was significantly related to poor OS while increased follicular helper $\mathrm{T}$ cell, plasma cell, and resting mast cell were related to better OS (on the verge of 


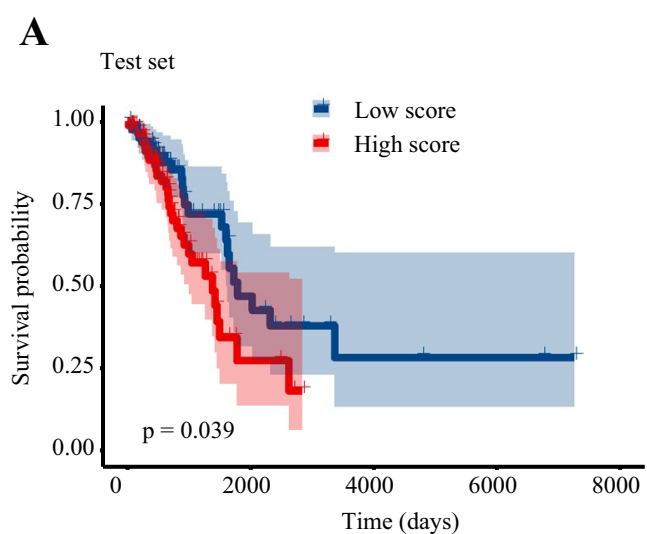

C

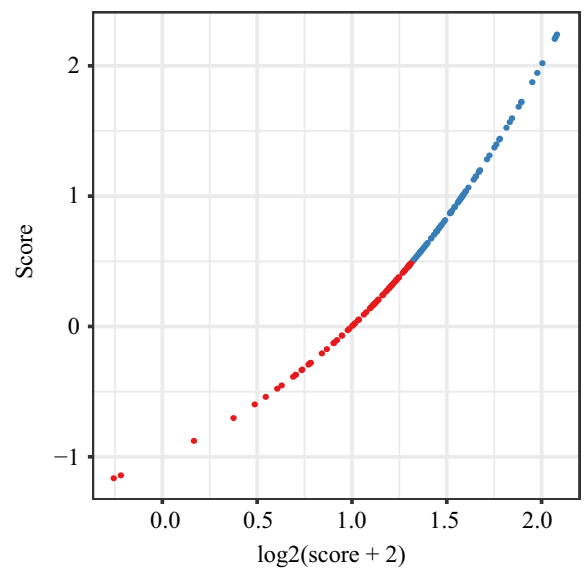

B



D

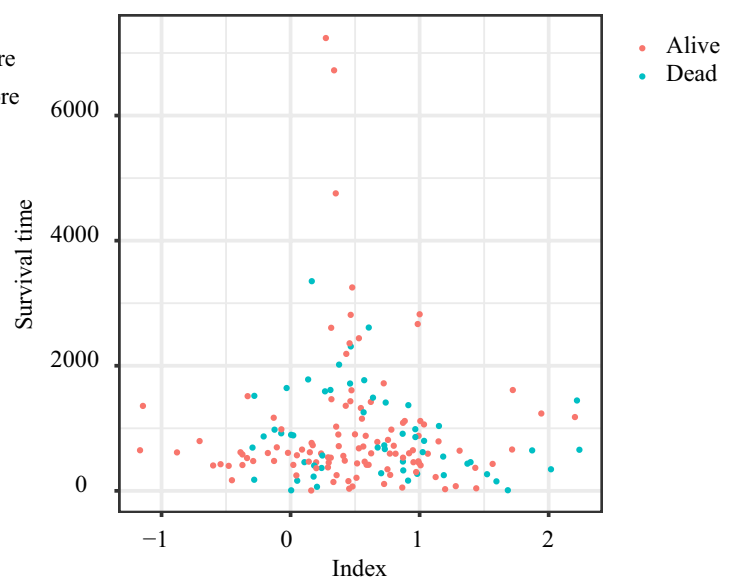

$\mathbf{E}$
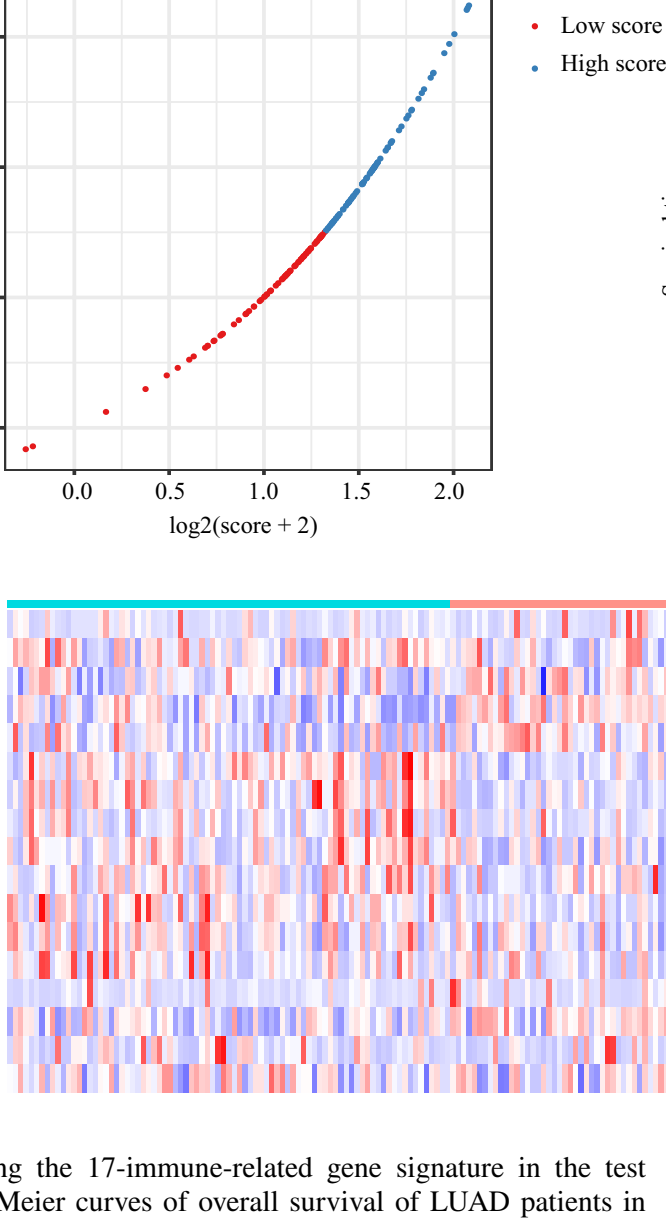

Fig. 4 Validating the 17-immune-related gene signature in the test set. a Kaplan-Meier curves of overall survival of LUAD patients in
high- and low-risk score groups. b Time-dependent receiver operat-

ing characteristic curves. $\mathbf{c}$ The distribution of risk scores. $\mathbf{d}$ The relationship between risk scores and survival times. e The expression patterns of 17 immune-related genes in high- and low-risk score groups

statistical significance, $p=0.058,0.15,0.20$, respectively) (Fig. 7b-e).

\section{The immune signature and TMB}

The mutation profiles of each LUAD patient were analyzed. For the total set, the top 20 most significantly mutated genes

were TTN, TP53, MUC16, RYR2, CSMD3, LRP1B, TNR, MUC17, CSMD1, ANK2, FAT3, ZNF536, NAV3, COL11A1, KRAS, XIRP2, SPTA1, FLG, ZFHX4, USH2A (Fig. 8a). Subsequently, we calculated the TMB of each sample and found TMB was markedly higher in the high-risk score group $(p=0.0020)$ (Fig. 8b). However, we observed that TMB was not related to patients' OS ( $p=0.75)$ (Fig. $8 \mathrm{c})$. 


\section{A}



C



B

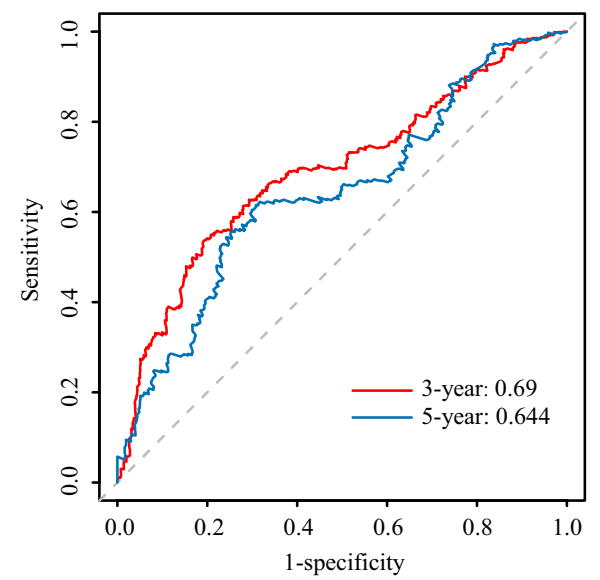

$\mathbf{D}$

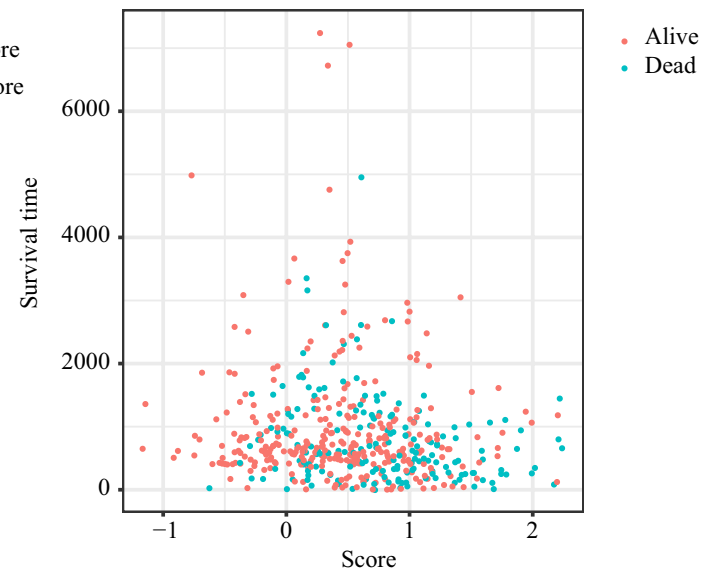

$\mathbf{E}$
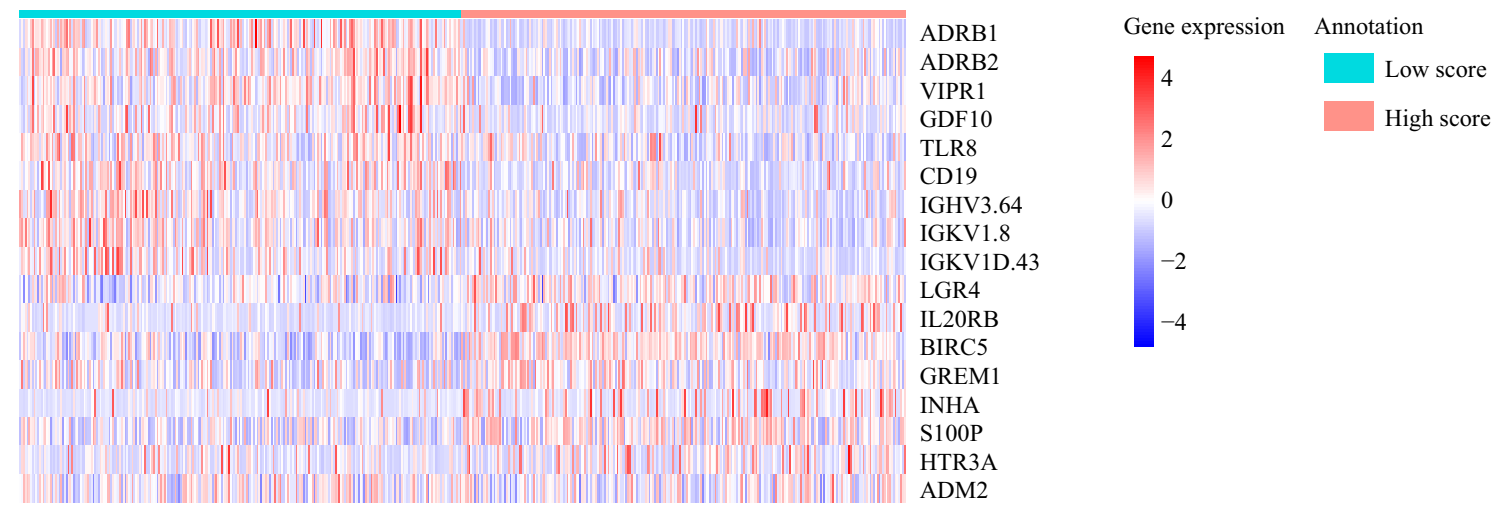

Fig. 5 Validating the 17-immune-related gene signature in the total dataset. a Kaplan-Meier curves of overall survival of LUAD patients in high- and low-risk score groups. b Time-dependent receiver operating characteristic curves. $\mathbf{c}$ The distribution of risk scores. $\mathbf{d}$ The relationship between risk scores and survival times. e The expression patterns of 17 immune-related genes in high- and low-risk score groups

ICI treatment was not available in TCGA LUAD dataset, we used two subtypes of IPS values (IPS-PD-1/PD-L1/ PD-L2_pos and IPS-CTLA-4_pos) as the surrogates of the LUAD patients' responses to anti-PD-1/PD-L1 and anti-CTLA-4 treatment. In this predictive model, the relative probabilities to respond to anti-PD-1/PD-L1 and
IPS is a machine learning-based scoring scheme, which could predict patients' response to immune checkpoint inhibitor in silico [23]. Given that the information on 
Table 2 Univariate and multivariate Cox regression analysis

\begin{tabular}{|c|c|c|c|c|}
\hline \multirow[t]{2}{*}{ Variables } & \multicolumn{2}{|l|}{ Univariate analysis } & \multicolumn{2}{|c|}{ Multivariate analysis } \\
\hline & $\mathrm{HR}(95 \% \mathrm{CI})$ & $P$ value & $\mathrm{HR}(95 \% \mathrm{CI})$ & $P$ value \\
\hline Age ( $\geq 65$ vs. $<65$ years $)$ & $1.09(0.809 \sim 1.47)$ & 0.57 & & \\
\hline T stage (T3-T4 vs. T1-T2) & $2.28(1.55 \sim 3.35)$ & $<0.0001$ & $1.7(1.06 \sim 2.8)$ & 0.029 \\
\hline $\mathrm{N}$ stage (N1-N3 vs. T0) & $2.64(1.96 \sim 3.56)$ & $<0.0001$ & $2.1(1.44 \sim 3.2)$ & $<0.0001$ \\
\hline M stage (M1 vs. M0) & $2.13(1.24 \sim 3.65)$ & 0.01 & $1.4(0.71 \sim 2.6)$ & 0.34 \\
\hline TNM stage (stage III-IV vs. I-II) & $2.63(1.93 \sim 3.59)$ & $<0.0001$ & $1.2(0.73 \sim 2.0)$ & 0.44 \\
\hline Risk score (high vs. low) & $2.52(1.85 \sim 3.43)$ & $<0.0001$ & $2.2(1.51 \sim 3.2)$ & $<0.0001$ \\
\hline
\end{tabular}

A

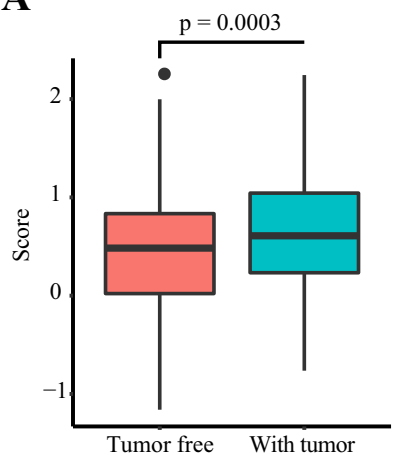

D



B

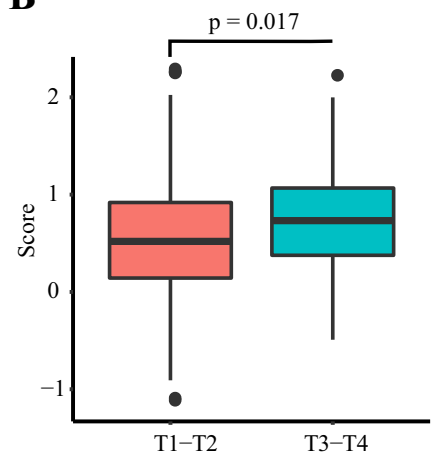

$\mathbf{E}$

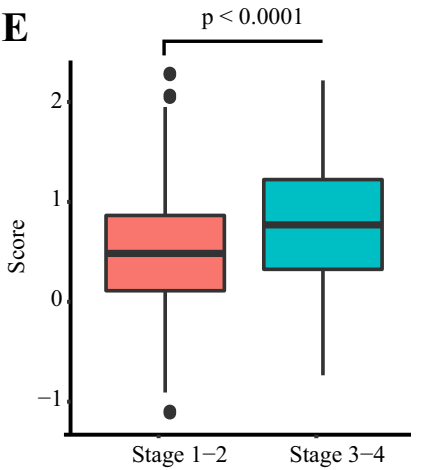

$\mathbf{C}$

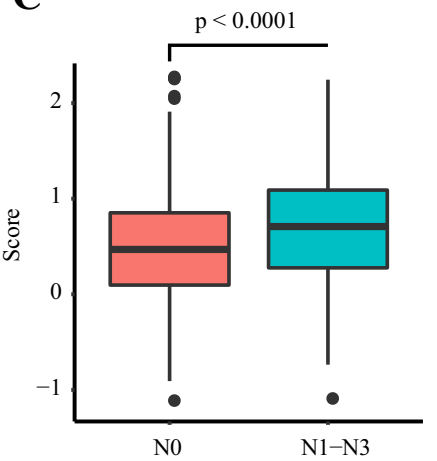

F

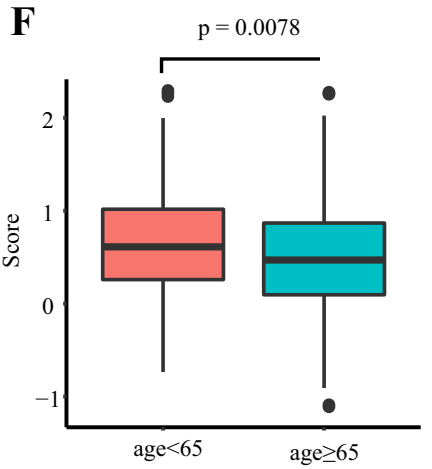

Fig. 6 The relationships between the immune signature and patients' clinic-pathologic parameters. a Tumor burden. b Tumor size. c Lymph node status. d Distant metastasis. e TNM stage. f Age at diagnosis

anti-CTLA-4 treatment were higher in the low-risk score group ( $p=0.0002$ and $p<0.0001$ ) (Fig. $8 \mathrm{~d}-\mathrm{e}$ ). The results indicated that patients with low immune signature score might be suitable for ICI treatment. Besides, we compared the expression levels of immune checkpoints and their ligands between the high-risk score group and low-risk score group (Fig. 8f-h). Patients with low-risk scores had modestly increased PD-L1 (mean expression level: 1.94 in the low-risk score group and 1.88 in the high-risk score group) and significantly elevated PD-1 $(p=0.002)$ and CTLA-4 $(p<0.0001)$.

\section{Discussion}

In the present study, we constructed a prognostic immune signature using TCGA LUAD dataset. This signature consisted of 17 IRGs. Among the 17 genes, it has been reported that $B I R C 5, S 100 P$, and $A D R B 2$ could preciously predict the outcomes of NSCLC patients [24-26]. For other IRGs, such as LGR4, GDF10, GREM1, IL2ORB, $I N H A, V I P R 1$, and $A D M 2$, they had been verified to participate in carcinogenesis and affect patients' prognoses in 




Fig. 7 The relationships between tumor-infiltrating immune cells and risk scores, as well as patients' overall survival. a The association between tumor-infiltrating immune cells and the immune risk signa-

ture. The associations between overall survival and (b) Macrophages (M0), (c) Follicular helper T cells, (d) Plasma cells, (e) Resting mast cells 


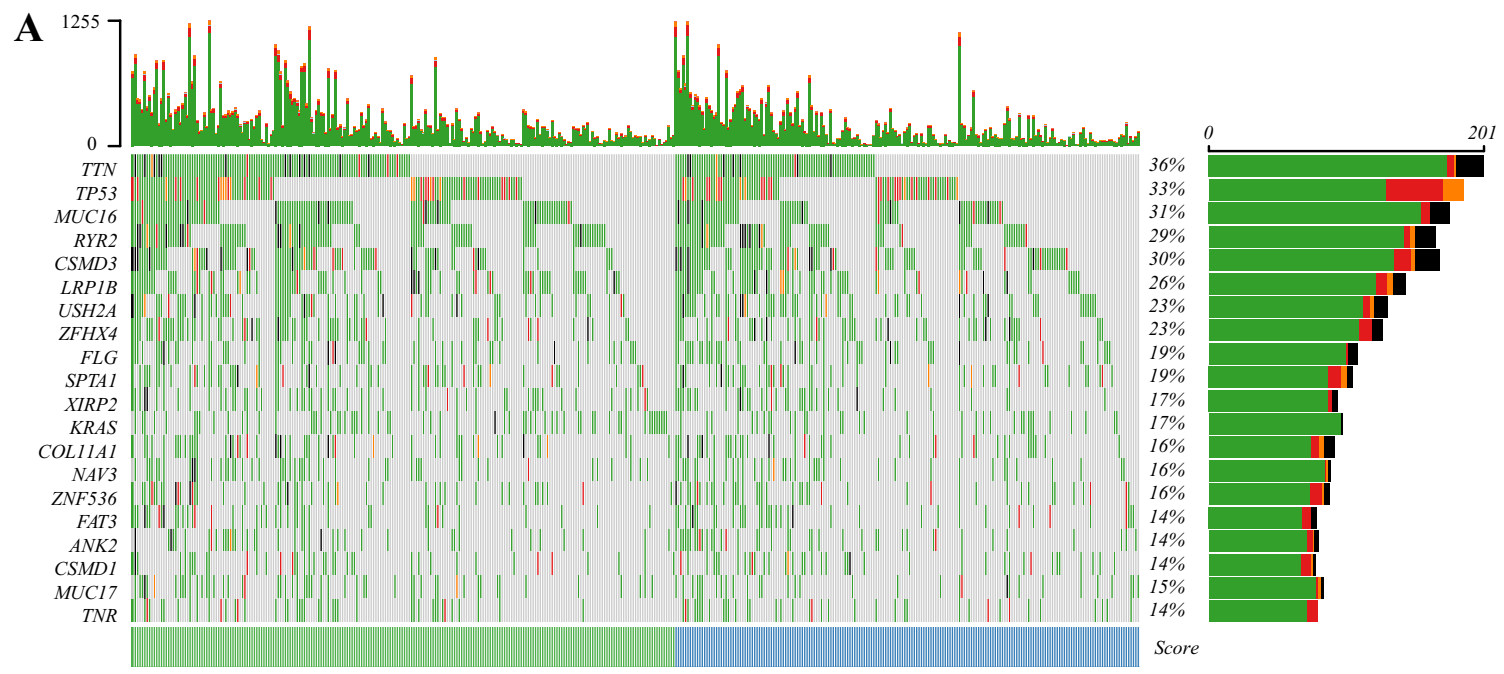

$\begin{array}{lll}\text { - Missense_Mutation } & \text { Translation_Start_Site } \\ \text { - } & \text { nonsense_Mutation } & \text { Multi_Hit }\end{array}$

- Splice Site

B



D

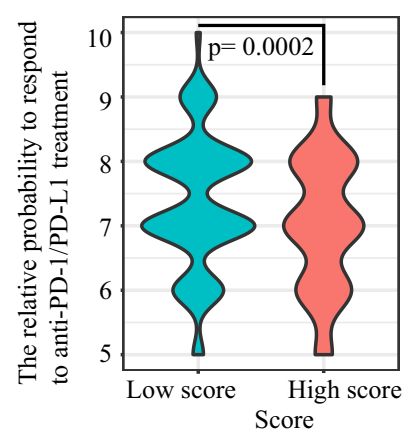

Score

High score $\quad$ Low score

C



E

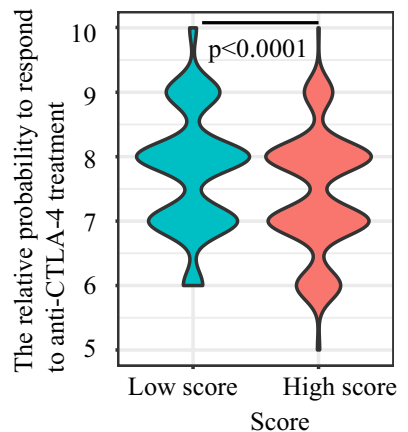



G

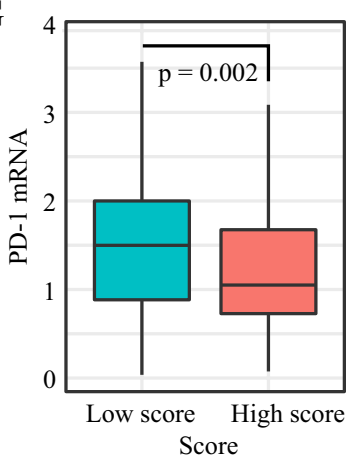

H




4Fig. 8 The mutation profile, TMB, relative probabilities to respond to immune checkpoint inhibitors, and the levels of immune checkpoint molecules in low-risk and high-risk groups. a Mutation profile of LUAD patients in low-risk and high-risk groups. b The difference of tumor mutation burden between low-risk and high-risk groups. c The association of tumor mutation burden and patients' overall survival. d-e The relative probabilities to respond to anti-PD-1/PD-L1 and anti-CTLA-4 treatment in the low-risk score and high-risk score group. The expressions of (f) PD-L1, (j) PD-1, and (h) CTLA-4 in low-risk and high-risk groups

other cancers, although the relevant studies were rare in lung cancer [27-34]. Notably, most selected IRGs could regulate cancer initiation and development by simultaneously modulating the status of the tumor immune microenvironment and the malignant biological properties of tumor cells [35]. For example, LGR4 (encoded by LGR4) and its ligands R-spo1-4 are not only a vital axis for tumor growth and metastasis but also promotes macrophage M2 polarization and tumor-associated macrophage (TAM) formation [35]. Besides, ADRB2-encoded protein $\beta 2$-adrenergic receptor ( $\beta 2$-AR) are widely expressed on activated and memory $\mathrm{CD} 8^{+} \mathrm{T}$ cells [36]. $\beta 2$-AR could mediate cancer immunosuppression by reprogramming the metabolism of activated T cells [36, 37]. Additionally, as an innate pattern recognition receptor, Toll-like receptor 8 (TLR8) (encoded by TLR8) could enhance cytokines secretion and promote anti-tumor immunity [38]. Activated TLR8 pathway remarkably reshapes the tumor immune microenvironment by decreasing infiltrating myeloid-derived suppressor cells (MDSCs), regulatory $\mathrm{T}$ cells (Tregs), and immunosuppressive markers, such as CTLA-4 [38]. Meanwhile, stimulating TLR8 signal could increase the numbers of tumor-infiltrating M1 monocytes and T cells [38].

Generally, previous predictive models for prognostic stratification focused on the intrinsic features of tumors, such as tumor size, lymph node metastasis, and distant metastasis. Actually, some elements of innate immunity and adaptive immunity actively participate in cancer development as well [39]. Effectors including cytotoxic T cells, B cells, and NK cells destroy tumor cells while Tregs, MDSCs, and TAMs can orchestrate immune escape and tumor growth [40]. A study in human colon cancer showed that the immunologic parameters (tumor-infiltrating immune cells' types, locations, and numbers) could more effectively predict patients' survival than traditional histopathological methods [41]. 'Immunoscore' is a quantitative forecasting tool based on tumor immune contexture which is under clinical studies in multiple cancer types as a supplement for the current histopathological staging system [42, 43]. Similar to 'Immunoscore,' our immune signature could also reflect the tumor immune microenvironment status and herald patients' survival based on RNA-sequencing data. Apart from patients' survival, this immune signature was also a predictor for patients' response to ICI treatment. Because the information on ICI treatment was not available in TCGA LUAD dataset, we used IPS as a surrogate for ICI treatment efficacy. IPS was developed mainly based on TCGA RNA-seq profiles, which can quantitatively predict patients' response to anti-PD-1/PD-L1 and anti-CTLA-4 therapies [23]. The IPS values were significantly higher in the low-risk score group, which indicated that this 17 -IRG signature might be useful for patient selection before ICI treatment. Up till now, PD-L1 expression, TMB, mismatch repair deficiency, microsatellite instability had been applied to select patients prior to ICI therapy [8]. In the present study, the predictive capability of this immune signature is independent of TMB. On the contrary, we found that the TMB was markedly lower in the low-risk score group. Considering that IPS is a complicated model containing multiple factors, we supposed that other variables, such as upregulated immune checkpoint signals, might contribute to the enhanced treatment effect in the low-risk score group.

According to the cancer-immunity cycle theory, anticancer immune response consists of multiple stepwise processes, including releasing antigens of cancer cells, captured and processed by antigen presentation cells, activation of $\mathrm{T}$ cells, trafficking and infiltrating of $\mathrm{T}$ cells into tumor, recognizing and killing cancer cells [44]. During cancer development, one or more steps are hampered, such as increased expressions of immune checkpoints and their ligands, impaired T cell infiltration, as well as antigenic modulation $[44,45]$. Therefore, in the condition that immune checkpoint is not the only rate-limiting step, patients might get little benefit from ICI treatment. In the present study, patients with low-risk scores had higher expressions of immune checkpoint molecules. Increased levels of immune checkpoints, such as PD- 1 and CTLA-4, indirectly indicated the preexisted $\mathrm{T}$ cell activation for the low-risk score group. Thus, patients with low-risk scores might be more sensitive to ICI treatment.

In spite of some positive results, some questions still remained. First, this immune signature was constructed based on public datasets. The predictive capability needs further verification in randomized controlled trials. Besides, we used the IPS value to mimic patients' response to ICI treatment. Although the correlation between IPS and response to ICI therapy had been validated in several independent datasets, IPS values still could not completely replace real treatment response.

\section{Conclusion}

We constructed a 17-IRG prognostic model to predict LUAD patients' survival and response to ICI treatment. Patients with low-risk scores had better prognosis and may be predicted to benefit with ICI therapy. This immune signature 
might be valuable for prognostic stratification and patient selection before ICI treatment. We believe this predictive model should be prospectively validated.

Acknowledgements Not applicable.

Author contributions MY performed the bioinformatics analysis, drafted the manuscript and prepared the figures. AL, LZ, and QC collected the related references and participated in discussion. SL and KW designed this review and revised the manuscript. All authors contributed to this manuscript. All authors read and approved the final manuscript.

Funding This work was supported by the National Natural Science Foundation of China (No. 81874120,82073370), Wuhan Science and Technology Bureau (No. 2017060201010170).

Availability of data and material TCGA LUAD gene expression profiles and patients' clinical data in this study are available at UCSC Xena (https://xena.ucsc.edu/). Gene mutation data could be acquired from TCGA data portal (https://portal.gdc.cancer.gov/). The patients' IPS values are available in the Cancer Immunome Atlas (https://tcia. at/home).

Code availability R software (4.0.0) could be downloaded from https ://www.r-project.org/. Some packages used in the study are available at Bioconductor (www.bioconductor.org/).

\section{Compliance with ethical standards}

Conflict of interest The authors declare that they have no competing interests.

Ethics approval Not applicable.

Consent for publication Not applicable.

Open Access This article is licensed under a Creative Commons Attribution 4.0 International License, which permits use, sharing, adaptation, distribution and reproduction in any medium or format, as long as you give appropriate credit to the original author(s) and the source, provide a link to the Creative Commons licence, and indicate if changes were made. The images or other third party material in this article are included in the article's Creative Commons licence, unless indicated otherwise in a credit line to the material. If material is not included in the article's Creative Commons licence and your intended use is not permitted by statutory regulation or exceeds the permitted use, you will need to obtain permission directly from the copyright holder. To view a copy of this licence, visit http://creativecommons.org/licenses/by/4.0/.

\section{References}

1. Siegel RL, Miller KD, Jemal A (2019) Cancer statistics, 2019. CA Cancer J Clin 69:7-34. https://doi.org/10.3322/caac.21551

2. Duma N, Santana-Davila R, Molina JR (2019) Non-small cell lung cancer: epidemiology, screening, diagnosis, and treatment. Mayo Clin Proc 94:1623-1640. https://doi.org/10.1016/j.mayoc p.2019.01.013
3. Denisenko TV, Budkevich IN, Zhivotovsky B (2018) Cell deathbased treatment of lung adenocarcinoma. Cell Death Dis 9:117. https://doi.org/10.1038/s41419-017-0063-y

4. Joseph NA, Chiou SH, Lung Z, Yang CL, Lin TY, Chang HW et al (2018) The role of HGF-MET pathway and CCDC66 cirRNA expression in EGFR resistance and epithelial-to-mesenchymal transition of lung adenocarcinoma cells. J Hematol Oncol 11:74. https://doi.org/10.1186/s13045-018-0557-9

5. Liu Q, Yu S, Zhao W, Qin S, Chu Q, Wu K (2018) EGFR-TKIs resistance via EGFR-independent signaling pathways. Mol Cancer 17:53. https://doi.org/10.1186/s12943-018-0793-1

6. Chen R, Manochakian R, James L, Azzouqa AG, Shi H, Zhang Y et al (2020) Emerging therapeutic agents for advanced non-small cell lung cancer. J Hematol Oncol 13:58. https://doi.org/10.1186/ s13045-020-00881-7

7. Wang Q, Yang S, Wang K, Sun SY (2019) MET inhibitors for targeted therapy of EGFR TKI-resistant lung cancer. J Hematol Oncol 12:63. https://doi.org/10.1186/s13045-019-0759-9

8. Yi M, Jiao D, Xu H, Liu Q, Zhao W, Han X et al (2018) Biomarkers for predicting efficacy of PD-1/PD-L1 inhibitors. Mol Cancer 17:129. https://doi.org/10.1186/s12943-018-0864-3

9. Yi M, Qin S, Zhao W, Yu S, Chu Q, Wu K (2018) The role of neoantigen in immune checkpoint blockade therapy. Exp Hematol Oncol 7:28. https://doi.org/10.1186/s40164-018-0120-y

10. Forde PM, Chaft JE, Smith KN, Anagnostou V, Cottrell TR, Hellmann MD et al (2018) Neoadjuvant PD-1 blockade in resectable lung cancer. N Engl J Med 378:1976-1986. https:// doi.org/10.1056/NEJMoa1716078

11. Li X, Shao C, Shi Y, Han W (2018) Lessons learned from the blockade of immune checkpoints in cancer immunotherapy. J Hematol Oncol 11:31. https://doi.org/10.1186/s1304 5-018-0578-4

12. Li T, Fan J, Wang B, Traugh N, Chen Q, Liu JS et al (2017) TIMER: a web server for comprehensive analysis of tumorinfiltrating immune cells. Cancer Res 77:e108-e110. https://doi. org/10.1158/0008-5472.can-17-0307

13. Newman AM, Liu CL, Green MR, Gentles AJ, Feng W, Xu Y et al (2015) Robust enumeration of cell subsets from tissue expression profiles. Nat Methods 12:453-457. https://doi.org/10.1038/nmeth .3337

14. Gide TN, Quek C, Menzies AM, Tasker AT, Shang P, Holst J et al (2019) Distinct Immune cell populations define response to Anti-PD-1 monotherapy and anti-PD-1/Anti-CTLA-4 combined therapy. Cancer Cell 35:238-255.e236. https://doi.org/10.1016/j. ccell.2019.01.003

15. Yang S, Wu Y, Deng Y, Zhou L, Yang P, Zheng Y et al (2019) Identification of a prognostic immune signature for cervical cancer to predict survival and response to immune checkpoint inhibitors. Oncoimmunology 8:e1659094. https://doi.org/10.1080/21624 02x.2019.1659094

16. Wu J, Zhao Y, Zhang J, Wu Q, Wang W (2019) Development and validation of an immune-related gene pairs signature in colorectal cancer. Oncoimmunology 8:1596715. https://doi. org/10.1080/2162402x.2019.1596715

17. Shen S, Wang G, Zhang R, Zhao Y, Yu H, Wei Y et al (2019) Development and validation of an immune gene-set based prognostic signature in ovarian cancer. EBioMedicine 40:318-326. https://doi.org/10.1016/j.ebiom.2018.12.054

18. Long J, Wang A, Bai Y, Lin J, Yang X, Wang D et al (2019) Development and validation of a TP53-associated immune prognostic model for hepatocellular carcinoma. EBioMedicine 42:363-374. https://doi.org/10.1016/j.ebiom.2019.03.022

19. Colaprico A, Silva TC, Olsen C, Garofano L, Cava C, Garolini D et al (2016) TCGAbiolinks: an R/Bioconductor package for integrative analysis of TCGA data. Nucleic Acids Res 44:e71. https://doi.org/10.1093/nar/gkv1507 
20. Bhattacharya S, Andorf S, Gomes L, Dunn P, Schaefer H, Pontius $\mathrm{J}$ et al (2014) ImmPort: disseminating data to the public for the future of immunology. Immunol Res 58:234-239. https://doi. org/10.1007/s12026-014-8516-1

21. Robinson MD, McCarthy DJ, Smyth GK (2010) edgeR: a Bioconductor package for differential expression analysis of digital gene expression data. Bioinformatics 26:139-140. https://doi. org/10.1093/bioinformatics/btp616

22. Mayakonda A, Lin DC, Assenov Y, Plass C, Koeffler HP (2018) Maftools: efficient and comprehensive analysis of somatic variants in cancer. Genome Res 28:1747-1756. https://doi.org/10.1101/ gr.239244.118

23. Charoentong P, Finotello F, Angelova M, Mayer C, Efremova M, Rieder D et al (2017) Pan-cancer immunogenomic analyses reveal genotype-immunophenotype relationships and predictors of response to checkpoint blockade. Cell Rep 18:248-262. https ://doi.org/10.1016/j.celrep.2016.12.019

24. Cao Y, Zhu W, Chen W, Wu J, Hou G, Li Y (2019) Prognostic value of BIRC5 in lung adenocarcinoma lacking EGFR, KRAS, and ALK mutations by integrated bioinformatics analysis. Dis Markers 9:5451290. https://doi.org/10.1155/2019/5451290

25. Kim B, Lee HJ, Choi HY, Shin Y, Nam S, Seo G et al (2007) Clinical validity of the lung cancer biomarkers identified by bioinformatics analysis of public expression data. Cancer Res 67:7431-7438. https://doi.org/10.1158/0008-5472.can-07-0003

26. Wang H, Hao B, Chen X, Zhao N, Cheng G, Jiang Y et al (2006) Beta-2 adrenergic receptor gene (ADRB2) polymorphism and risk for lung adenocarcinoma: a case-control study in a Chinese population. Cancer Lett 240:297-305. https://doi.org/10.1016/j. canlet.2005.09.018

27. Yue Z, Yuan Z, Zeng L, Wang Y, Lai L, Li J et al (2018) LGR4 modulates breast cancer initiation, metastasis, and cancer stem cells. Faseb J 32:2422-2437. https://doi.org/10.1096/fj.20170 0897R

28. Zhou T, Yu L, Huang J, Zhao X, Li Y, Hu Y et al (2019) GDF inhibits proliferation and epithelial-mesenchymal transition in triple-negative breast cancer via upregulation of Smad7. Aging Albany NY 11:3298-3314. https://doi.org/10.18632/aging.101983

29. Ren J, Smid M, Iaria J, Salvatori DCF, van Dam H, Zhu HJ et al (2019) Cancer-associated fibroblast-derived Gremlin 1 promotes breast cancer progression. Breast Cancer Res 21:109. https://doi. org/10.1186/s13058-019-1194-0

30. Haider S, Wang J, Nagano A, Desai A, Arumugam P, Dumartin L et al (2014) A multi-gene signature predicts outcome in patients with pancreatic ductal adenocarcinoma. Genome Med 6:105. https ://doi.org/10.1186/s13073-014-0105-3

31. Yoon W, Yoo Y, Chae YS, Kee SH, Kim BM (2018) Therapeutic advantage of genetically engineered Salmonella typhimurium carrying short hairpin RNA against inhibin alpha subunit in cancer treatment. Ann Oncol 29:2010-2017. https://doi.org/10.1093/ annonc/mdy 240

32. Wu FQ, Fang T, Yu LX, Lv GS, Lv HW, Liang D et al (2016) ADRB2 signaling promotes HCC progression and sorafenib resistance by inhibiting autophagic degradation of HIF1 $\alpha$. J Hepatol 65:314-324. https://doi.org/10.1016/j.jhep.2016.04.019

33. Xu B, Lv W, Li X, Zhang L, Lin J (2019) Prognostic genes of hepatocellular carcinoma based on gene coexpression network analysis. J Cell Biochem. https://doi.org/10.1002/jcb.28441

34. Hollander LL, Guo X, Salem RR, Cha CH (2015) The novel tumor angiogenic factor, adrenomedullin-2, predicts survival in pancreatic adenocarcinoma. J Surg Res 197:219-224. https://doi. org/10.1016/j.jss.2014.11.002

35. Tan B, Shi X, Zhang J, Qin J, Zhang N, Ren H et al (2018) Inhibition of Rspo-Lgr4 facilitates checkpoint blockade therapy by switching macrophage polarization. Cancer Res 78:4929-4942. https://doi.org/10.1158/0008-5472.can-18-0152

36. Qiao G, Bucsek MJ, Winder NM, Chen M, Giridharan T, Olejniczak SH et al (2019) $\beta$-Adrenergic signaling blocks murine CD8(+) T-cell metabolic reprogramming during activation: a mechanism for immunosuppression by adrenergic stress. Cancer Immunol Immunother 68:11-22. https://doi.org/10.1007/s0026 2-018-2243-8

37. Daher C, Vimeux L, Stoeva R, Peranzoni E, Bismuth G, Wieduwild $\mathrm{E}$ et al (2019) Blockade of $\beta$-adrenergic receptors improves CD8(+) T-cell priming and cancer vaccine efficacy. Cancer Immunol Res 7:1849-1863. https://doi.org/10.1158/2326-6066. cir-18-0833

38. Shayan G, Kansy BA, Gibson SP, Srivastava RM, Bryan JK, Bauman JE et al (2018) Phase Ib study of immune biomarker modulation with neoadjuvant cetuximab and TLR8 stimulation in head and neck cancer to overcome suppressive myeloid signals. Clin Cancer Res 24:62-72. https://doi.org/10.1158/1078-0432. ccr-17-0357

39. Gajewski TF, Schreiber H, Fu YX (2013) Innate and adaptive immune cells in the tumor microenvironment. Nat Immunol 14:1014-1022. https://doi.org/10.1038/ni.2703

40. Taube JM, Galon J, Sholl LM, Rodig SJ, Cottrell TR, Giraldo NA et al (2018) Implications of the tumor immune microenvironment for staging and therapeutics. Mod Pathol 31:214-234. https://doi. org/10.1038/modpathol.2017.156

41. Galon J, Costes A, Sanchez-Cabo F, Kirilovsky A, Mlecnik $B$, Lagorce-Pagès C et al (2006) Type, density, and location of immune cells within human colorectal tumors predict clinical outcome. Science 313:1960-1964. https://doi.org/10.1126/scien ce. 1129139

42. Angell H, Galon J (2013) From the immune contexture to the Immunoscore: the role of prognostic and predictive immune markers in cancer. Curr Opin Immunol 25:261-267. https://doi. org/10.1016/j.coi.2013.03.004

43. Pagès F, Mlecnik B, Marliot F, Bindea G, Ou FS, Bifulco C et al (2018) International validation of the consensus Immunoscore for the classification of colon cancer: a prognostic and accuracy study. Lancet 391:2128-2139. https://doi.org/10.1016/s0140 -6736(18)30789-x

44. Chen DS, Mellman I (2013) Oncology meets immunology: the cancer-immunity cycle. Immunity 39:1-10. https://doi. org/10.1016/j.immuni.2013.07.012

45. Mariathasan S, Turley SJ, Nickles D, Castiglioni A, Yuen K, Wang Y et al (2018) TGF $\beta$ attenuates tumour response to PD-L1 blockade by contributing to exclusion of T cells. Nature 554:544 548. https://doi.org/10.1038/nature25501

Publisher's Note Springer Nature remains neutral with regard to jurisdictional claims in published maps and institutional affiliations. 